\title{
Effect of doping on the optical gain and the spontaneous noise enhancement factor in quantum well amplifiers and lasers studied by simple aralytical expressions
}

\author{
Kerry $\rfloor$. Vahala \\ 128-95 California Institute of Technology. Pasadena, California 91125 \\ C. E. Zah \\ Bell Communications Research, Red Bank, New Jersey 07701-7020
}

(Received 14 March 1988; accepted for publication 11 April 1988)

\begin{abstract}
The maximum optical gain and the spontaneous noise enhancenent factor in quantum well structures are expressed as extremely simple functions that are accurate over a wide range of cartier densities. These expressions are used to study the efect of doping on the optical gain and the noise enhancement factor in a $100 \mathrm{~A}$ InGaAs/In quantum well stracture. n-type doping is most effective in reducing the transparency exciation level (laser threshold) and the noise enhancement factor (amphifer noise fgure), whereas $p$-type doping enables increased gain at a given excitation level.
\end{abstract}

Quantum well material is now prepared routinely in several compound semiconductor systems. The semiconductor laser has benefted greatly from the development of these structures. Lasers employing the appropriately designed single or multiple quantum well active layers exhibit lower threshold current densities, reduced phase noise, and inproved dynamic response as compared to their conventional counterparts. ${ }^{1-4}$ Optical amplifiers employing quantum woll active layers should also exhbit improved performance. ${ }^{5}$ To optinize a quantum well active layer for improved device performance, it is desirable to have simple expressions for optical gain and related quantities as a function of excitation, doping, well widh, temperature, etc. In this letter we will show that very simple approximate expressions for optical gain, spontaneous emission, and the spontaneous noise enhancement factor can be derived for quantum well structures. These expressions inciude the effects of multiple subbands and remain accuiate for excitation levels that result in degenerate carrier populations. As an application of these expressions, we use them to investigate the efect of $p$-and $n$ type doping on gain and the noise enhancement factor in a $100 \mathrm{~A}$ InGaAs/InE quantum well structure.

We assume throughout this analysis that all subbands are parabolic and that optical transitions obey rigorous $k$ selection rules. Transition broadening is neglected (i.e., $T_{2}$ $=\infty$ ). We focus on gain resulting from the lowest energy subband in the conduction and valenee bands. The point of maximum gain in this case always occurs for the band-edge transitions. (In the bulk the gain peak lies above the band edge and depends strongly on excitation.) Under these assumptions the maximum optical gain $G_{\max }$ and the spontaneous noise $S$ at the gain peak are given by the following expressions:

$$
\begin{aligned}
& G_{\max }=G_{0}\left[f_{c}(n)-f_{0}(n)\right], \\
& S=G_{0} f_{c}(n)\left[1-f_{0}(n)\right] .
\end{aligned}
$$

$G_{0}$ is a constant given by

$$
G_{0}=E_{0}|\mu|^{2} m^{*} N / \epsilon T^{3} W
$$

where $E_{0}$ is the transition energy, $\mu$ is the dipole moment matrix clement for the transition, $m *$ is the reduced mass, $N$ is the number of quantum weils interacting with the optical field, $\epsilon$ is the dielectric constant of the material, and $W$ is the effective width of the optical mode." This $G_{0}$ includes the effect of incomplete spatial overlap of the optical mode with the quantur wells (i.e., the confinement factor is inciuded). The gain constant without the spatial overlap integral in chuded (the "material" gain) is found by replacing $N / W$ with $1 / L_{z}$. Equation (3) is most easigy applied in separate confinement structures. In these structures, the effective width $W$ is approximately independent of the number of quantum wells, $N$. The units of $G_{0}$ are inverse rime.

The quantities $f_{c}(n)$ and $f_{n}(n)$ are Fermi electronic occupancies for states at the lowest energy conduction and valence subband colges, respectively. We assume quasineutralty so that each occuparcy is determined by knowledge of the electron density. By straightorward integration of the quasi-Femi occupancy over states in the conduction band, followed by substitution using $f$, the following relation is established between $n$ and $f_{c}$,

$$
n=\sum_{i=0} n_{i} \log \left(1+\frac{f_{c}}{1-f_{s}} e^{-\epsilon_{c l}}\right)
$$

where the summation is over subbands with the / th subband edge located $\epsilon_{s}$ (in units of $\kappa_{B} T$ ) higher in energy than the lowest energy subband edge (i.e., $\left.\epsilon_{c 0}=0\right)$. The quantites $n_{1}$ are given by

$$
n_{l}=\kappa_{B} T m_{c l} / \pi h^{2} L_{z}
$$

where $m_{c l}$ is the efiective mass of the $l$ th conduction subband and $L_{z}$ is the well width. An equation similar to (4) holds for the valence bands with $f_{c}$ replaced by $1-f_{i}, \epsilon_{c l}$ replaced by $\left|\epsilon_{n}\right|$, and $n$, replaced by $p_{l}$ with corresponding valence-band effective rasses entering into Eq. (5). To rewrite Esss. (1) and (2) in terms of carrier density, it is necessary to invert Eq. (4) and the corresponding equation for the valence band. For the case of bulk material the analog of Eq. (4) is not a closed form expression and its approximate inverted form must be derived using the Joyce-Dixon approximation. For guantum wells we have found a very simple inversion formula. It can be verifled by direct substivtion that Eq. (4) and the corresponding valence-band equation have 
the following approximate inverted forms:

$$
\begin{aligned}
& f_{c}=1-e^{-n f y}, \\
& f_{v}=e^{-n / s},
\end{aligned}
$$

where, for example,

$$
\mathscr{N}=\sum_{i=0} n_{l} e^{-\cdots \epsilon_{c !}}
$$

The validity condition for this approximation is given below for Eq. (7). Equation (6) has a similar validity condition which is always satisfied provided the condition on (7) is valid. The validity condition is

$$
\frac{\Sigma_{l=1} p_{l} e^{-\epsilon_{v l}}\left(1-e^{-\epsilon_{u}}\right)}{2 \mathscr{P}^{2}} p \ll 1
$$

This expression demonstrates an interesting property of this approximation. If we consider varying the subband energy spacings simultaneously (say by varying the well width), then Eq. (9a) suggests that there is no error to second order at the extremes. In fact, it can be shown that the results in (6) and (7) are exact at these endpoints to the extent that we can still use the effective mass approximation for ulranarrow wells. If we neglect the second exponential in the sum, then $(9 a)$ has the following more useful form:

$$
\frac{\mathscr{P}-p_{0}}{2 \mathscr{P}^{2}} p \ll 1 \text {. }
$$

As an example, consider a $100 \AA$ quantum well in the InGaAs/InP system. We use $m_{c}=0.041 m_{0}$ and assume all heavy-hole-related subbands have effective masses $m_{\mathrm{hh}}=0.5 m_{0}$ and all light-hole-related subbands have effective masses $m_{1 h}=0.051 m_{0}$ (i.e., band mixing effects are neglected) ${ }^{8}$ It is sufficient to consider only the first conduction subband and the four lowest valence subbands in our calculation of $\mathscr{P}$ and $\mathscr{P}$. At room-temperature condition $(96)$ gives $p \ll 4.7 \times 10^{19} \mathrm{~cm}^{-3}$. The approximation is therefore valid over an extremely wide range of carrier densities.

As a further test of the approximation, we have plotted $f_{c}$ and $f_{v}$ versus carrier density at several donor (Fig. 1 ) and acceptor (Fig. 2) concentrations levels using Eqs. (6) and (7) and the exact form for comparison. There is only a slight deviation from the exact result for the case of heavy $p$ doping. These plots are interesting in one other respect. From $\mathrm{Eq}$. (1) the intersection of $f_{c}$ and $f_{b}$ corresponds to the transparency excitation level. Therefore, plotting the exponential

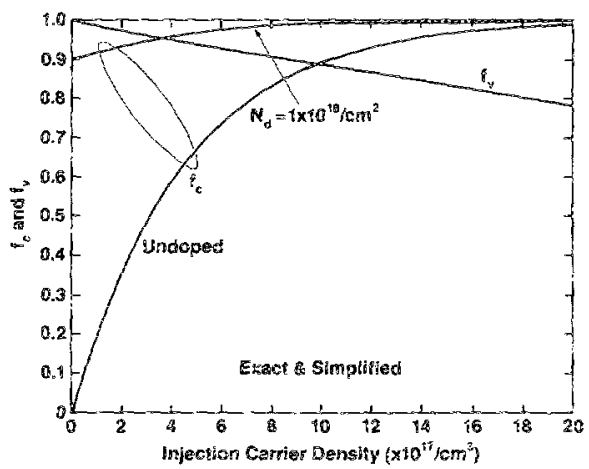

FlG. 1. Approximate (solid curve) and exach (dashed curve) plots of $f_{\mathrm{c}}$ and $f_{v}$ vs carrier density at several donor concentration levels for a $100 \AA$ InGaAs/Iny quantum well. Sold and dashed curves are coincident.

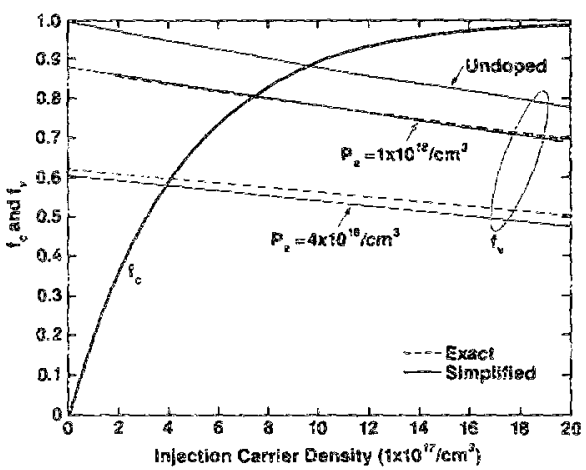

FIG. 2. Approximate (solid curve) and exact (dashed curve) plots of $f_{c}$ and $f_{0}$ vs carrier density at several aceptor concentration leveis for a $j 00 \AA$ InGaAs/hin quantum well.

functions in (6) and (7) in this way leads to a very simple graphical means of estimating transparency shifts under various conditions. Notice that the inclusion of donors or acceptors merely shifts the appropriate curve along the horizontal axis by the amount of doping level. In expressions (6) and (7) and all that follow, the presence of donors or acceptors can be accounted for by making the following replacements:

donors: $n / \mathscr{N} \rightarrow\left(n+N_{D}\right) / \mathscr{N}$,

acceptors: $n / \mathscr{P} \rightarrow\left(n+N_{A}\right) / \mathscr{P}$.

Finally, we substitute Eqs. (6) and (7) into Eqs. (1) and (2) to obtain the following expressions:

$$
\begin{aligned}
& G_{\max }=G_{0}\left(1-e^{-n / H}-e^{-n / \mathscr{P}}\right), \\
& \frac{d G_{\max }}{d n}=G_{0}\left(\frac{1}{\mathscr{N}} e^{\cdots n / H}+\frac{1}{\mathscr{P}} e^{-n / \mathscr{P}}\right), \\
& S=G_{0}\left(1-e^{-n / M}\right)\left(1-e^{-n / \mathscr{H}}\right), \\
& n_{\mathrm{sp}}=\frac{S}{G_{\max }}=1+\frac{e^{-n / H} e^{-n / \mathscr{P}}}{1-e^{-n / H}-e^{-n / \mathscr{P}}} .
\end{aligned}
$$

$n_{\mathrm{sp}}$ is the amplifier noise enhancement factor (also called the spontaneous emission factor in semiconductor lasers). We have also given an expression for the differential gain in (106). This quantity plays a central role in determining the fundamental frequency response of semiconductor lasers. We consider again the case of a $100 \AA$ quantum well in the InGaAs/InP system. $G_{\max }$ per quantum well for TE modes and $n_{\mathrm{sp}}$ are plotted versus carrier density in Figs. 3 and 4 at various donor and acceptor concentration levels. The $G_{\max }$ of a multiple quantum well separate confinement structure is

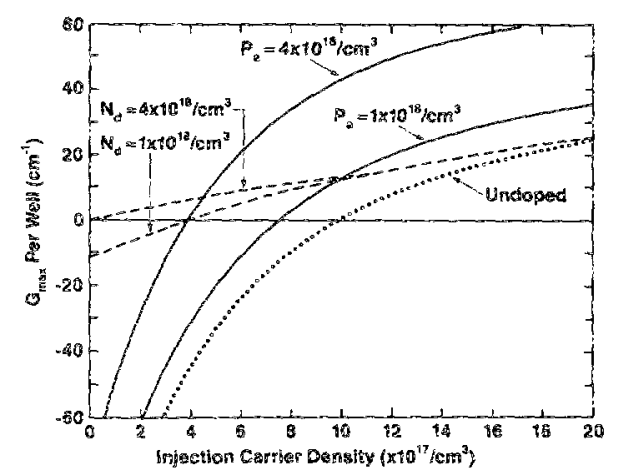

FIG. 3. Optical gain per quantum well vs carrier density at various donor and acceptor concentration levels for a $100 \AA$ InCaAs/AnP quantum well. 


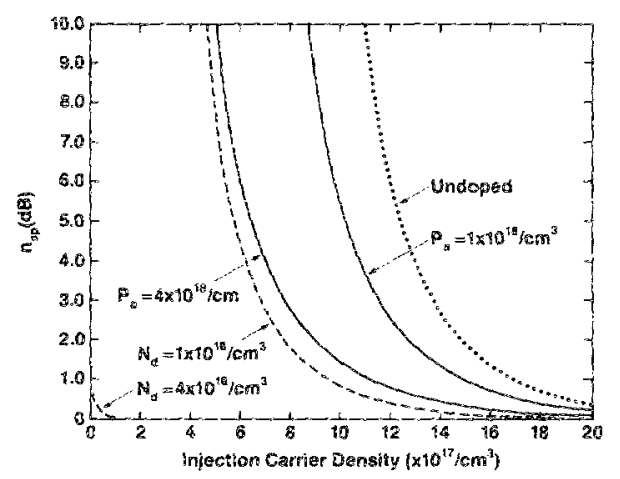

FIO. 4. Spontaneous noise enhancement factor vs carrier density at various donot and acceptor concentration levels for a $100 \mathrm{~A}$ In GaAs/In quanum well.

found by multiplying the values in Fig. 3 by the number of quantum wells. In the calculation of $G_{\max }$ we have assumed a

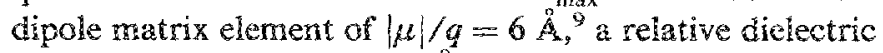
constant of $12.7, W=3000 \AA$, and a lasing wavelength of $1.55 \mathrm{fm}$. In Fig. 3 it is interesting to note that both $p$ and $n$ type doping lead to increased gain and decreased transparency levels as compared to the intrinsic case. In general, however, the magnitude of $G_{\max }$ is more strongly infuenced by p-type doping, whereas the transparency level is more strongly influenced by $n$-type doping. In Fig. $4, n_{\mathrm{sp}}$ at a given excitation level is reduced for beth $p$ - and $n$-type doping. $n$ type doping is more effective in reducing $n_{\text {sp }}$, however, reflecting the more rapid reduction in transparency excitation level exhibited in Fig. 3. These same conclusions apply in other material systems having a strong conduction band/valence band effective mass asymmetry. In particular, nearly identical results to those in Figs. 3 and 4 hold in the GaAs(AlGaAs) system.

Based on these results we propose that $n$-type modulation doping of quantum well active layers may have certain benefts to lasers and amplifers. First, the ability to recuce the transparency excitation level when combined with a reduction in the overall laser cavicy losses coud reduce laser thresholds beyond the already impressive limits that have been set in quantum well lasers. ${ }^{3}$ Second, $n$-rype modulation doping in a quantum well amplifier could significantiy improve the noise figures of these devices (note: $N F \approx 2 n_{s p}$ ). For the same reason laser intensity noise and phase noise will also be reduced. Note that, in general, operation at wavelengths shorter than the gain peak wavelength whll result in slightly larger $n_{\mathrm{sp}}$ values.

The potential advantage of $p$-type doping for laser operation is increased modulation speed. Wh Fig. 5 the differential material gain normalized by the bulk material value for InGaAs has been ploted. The intrinsic direct modulation speed varies as the square root of the differential gain at the operating point carrier density and wavelength. Note that in Fig. 5 the differential gain of the quantum well is, in fact, reduced by doping of the quantum well, thus rending to reduce direct modulation speed at a given operating point. $p$ type doping causes a minor reduction, and $n$-type doping causes a large reduction. The signt $p$-type-induced reduction can be offet, however, by the ability to operate at a lower carrier density since transparency is also reduced.

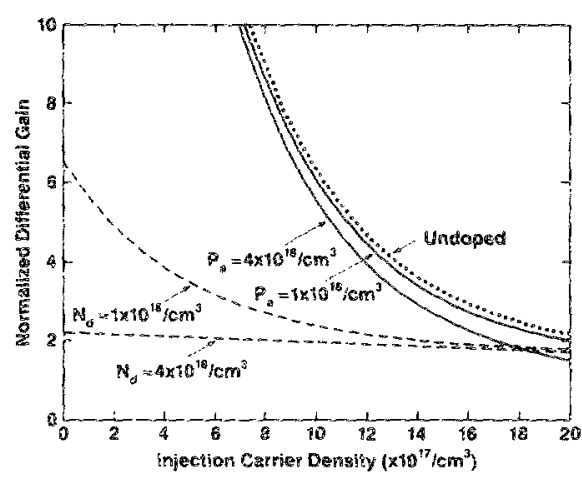

FIO. 5. Quantum well differential gain normalized by the typical value for bulk differential gain in the InGaAs system.

This idea has been demonstrated experimentally using $p$. type molulation-doped guantum well active layers. ${ }^{4}$

In conclusion, we have derived very simple and accurate expressions for the optical gain and the spontaneous emission noise factor of a quantum well active layer. The effect of varying excitation level, quantum well widh, donor or acceptor concentration levels, and temperature can be rapidly tested using these expressions. As an example, we have used the expressions to calculate maximum gain and the spontaneous noise factor versus carrier density in a $100 \AA$ InGaAs/InP quantum well. We noted that p-type doping is more effective in increasing gain, whereas $n$-type doping is more effective in reducing the transparency excitation level and $n_{\mathrm{sp}}$. The simple formulas developed here do not include the effects of intervalence band absorption, and therefore certain predictions, particularly those pertaining to gain in $p$-type longer wavelength systems, may be only qualitatively correct. Furthermore, all results have been expressed in terms of carrier densities. To relate these to an injection current requires knowledge of carrier recombination rates. In the longer wavelength systems these will have a strong Auger component which could diminish the improvements derived by heavy doping. The results, however, should be unaffected in the shorter wavelength systems. We plan to investigate the efiect of intersubband absorption and Auger recombination on the predictions of this approximation elsewhere. Finaly, we note that the calculation of 9 employed here used the bulk values for heavy and light hole effective masses. To be more accurate one could use the in-plane effective masses found by properly accounting for valence-band mixing effects by the quantum well.

The authors are grateful for severa! stimulating discussions with Tien Pei Lee.

'Y. Arakawa, K. Vahala, and A. Yuriv, Appl. Phys. Lett. 45, 950 (1984). 'S. Noda, K. Kojima, K. Kyuma, K. Hamanaka, and T. Nakayama, Appl. Phys. Lett. 50, 863 (1987).

P. I. Derry, A. Yariv, K. Y. Lau, N. Bar-Chaim, K. Lee, and J. Rosenberg, Appl. Phys. Lett. 50, $1773(1987)$.

${ }^{4} \mathrm{~K}$. Uomi, T. Mishima, and N. Chirone, Appi. Phys. Lett. 31,78 (1987).

${ }^{5} \mathrm{~K}$. Vahala and Chung-en $Z$ ah (unpublished).

6. Vahala and A. Yariv, Phys. Rev. A 32,345 (1985).

7W. B. Joyce and R. W. Dixon, Appl. Phys. Leit. 31, 354 (1977).

${ }^{8}$ F. Fiedler and A. Schlachetzki, Solid-State Electron. 30, 73 (1987).

${ }^{9} M$. Asada, A. Kancyama, and $Y$. Suematsu, J. Quantum Electron. QE-20, 745 (1984) 